\title{
Dynamic network participation of functional connectivity hubs assessed by resting-state fMRI
}

\author{
Alexander Schaefer ${ }^{1 *}$, Daniel S. Margulies ${ }^{2}$, Gabriele Lohmann $^{3}$, Krzysztof J. Gorgolewski $^{2}$, \\ Jonathan Smallwood ${ }^{4}$, Stefan J. Kiebel ${ }^{1,5}$ and Arno Villringer ${ }^{1,6,7,8}$ \\ 1 Department of Neurology, Max Planck Institute for Human Cognitive and Brain Sciences, Leipzig, Germany \\ ${ }_{2}^{2}$ Max Planck Research Group for Neuroanatomy \& Connectivity, Max Planck Institute for Human Cognitive and Brain Sciences, Leipzig, Germany \\ ${ }^{3}$ Department of High-field Magnetic Resonance, Max Planck Institute for Biological Cybernetics, Tübingen, Germany \\ ${ }^{4}$ Department of Psychology, The University of York, Hesslington, UK \\ ${ }^{5}$ Department of Neurology, Biomagnetic Center, University Clinics Jena, Jena, Germany \\ ${ }^{6}$ Berlin School of Mind and Brain, Mind and Brain Institute, Berlin, Germany \\ 7 Department of Cognitive Neurology, University Hospital Leipzig, Leipzig, Germany \\ ${ }^{8}$ Center for Stroke Research, Charité - Universitätsmedizin, Berlin, Germany
}

\section{Edited by:}

Shuhei Yamaguchi, Shimane

University, Japan

Reviewed by:

Qingbao Yu, The Mind Research

Network, USA

Carsten Giessing, Carl von

Ossietzky University Oldenburg,

Germany

R. Matthew Hutchison, Western

University, Canada

*Correspondence:

Alexander Schaefer, Max Planck Institute for Human Cognitive and Brain Sciences, Stephanstrasse 1a, 04103 Leipzig, Germany

e-mail:aschaefer@cbs.mpg.de
Network studies of large-scale brain connectivity have demonstrated that highly connected areas, or "hubs," are a key feature of human functional and structural brain organization. We use resting-state functional MRI data and connectivity clustering to identify multi-network hubs and show that while hubs can belong to multiple networks their degree of integration into these different networks varies dynamically over time. The extent of the network variation was related to the connectedness of the hub. In addition, we found that these network dynamics were inversely related to positive self-generated thoughts reported by individuals and were further decreased with older age. Moreover, the left caudate varied its degree of participation between a default mode subnetwork and a limbic network. This variation was predictive of individual differences in the reports of past-related thoughts. These results support an association between ongoing thought processes and network dynamics and offer a new approach to investigate the brain dynamics underlying mental experience.

Keywords: graphs, self-generated thoughts, brain networks, mind wandering

\section{INTRODUCTION}

One of the computational principles underlying behavior is that neuronal networks interact in a highly dynamic fashion (Dickinson, 1995; Marder and Bucher, 2001, 2007). While single neurons have been found to participate in multiple networks by means of the modulation of their synaptic connectivity (Hooper and Moulins, 1989; Weimann and Marder, 1994), it is an open question whether these dynamic events have an equivalent at the macroscopic, interregional level. Recent neuroimaging research in humans supports this hypothesis by demonstrating correspondence between large-scale brain networks and EEG microstates (Britz et al., 2010; Musso et al., 2010), which are transient, quasistable patterns in the EEG signal (Musso et al., 2010); as well as varying correlations between regions in the default-mode and task-positive networks (Chang and Glover, 2010). The existence of different large-scale brain states (Smith et al., 2012; Allen et al., 2014) suggests a spatially overlapping organization of specific areas. It is therefore conceivable that the dynamics of these regions allow brain areas to be members of multiple networks by varying their degree of membership over time.

One question facing cognitive neuroscience is how the full repertoire of cognitive capacities can be managed in a flexible manner. The finding of dynamic connectivity raises the possibility that temporal changes in brain connectivity may influence both mental states and behavior (Hutchison et al., 2013b; Allen et al.,
2014). For example, the observation of a relationship between connectivity dynamics and heart rate variability provides support for an association to the current mental state (Chang et al., 2013). Other work has demonstrated that dynamic physiological measures such as pupillometry (Smallwood et al., 2012), the electroencephalogram (Barron et al., 2011), and changes in fMRI (Christoff et al., 2009; Smallwood et al., 2013) have all been linked to variations in mental state. A recent study found that alterations in current task performance are predicted by the extent of anti-correlation between the average signal of networks shortly preceding the task (Thompson et al., 2013). Furthermore, the flexibility of functional network configuration during a learning task has been shown to be predictive of later learning performance (Bassett et al., 2011). Given these findings, the hypothesis is that the dynamic interplay of different brain networks modulates ongoing thoughts or the current mental state. Ongoing thoughts during the resting-state can be assessed by a subsequent introspective self-report. Here, we want to examine if there is a relation between ongoing dynamics of functional connectivity and later self-reported thoughts.

Highly connected brain areas or hubs, which can be detected using structural (Hagmann et al., 2008; Gong et al., 2009) and functional (Buckner et al., 2009; Lohmann et al., 2010; Zuo et al., 2012) neuroimaging, have been shown to play a central role in whole brain communication 
(Sporns et al., 2007; Van den Heuvel and Sporns, 2013). Here, we hypothesized that multi-network hubs at the intersection of different networks may serve as dynamic relay stations to support communication between these networks as indicated by animal studies (Dickinson, 1995; Marder and Bucher, 2001, 2007). To examine this dynamic hypothesis, we tested if multi-network hubs keep their participation in each network at a constant level over time or rather dynamically change their degree of membership. We applied an edge clustering approach (Ahn et al., 2010) to cluster connectivity itself, thereby allowing regions to participate in multiple networks. The advantage of using this connectivity clustering algorithm is that we can directly assess the dynamically changing degree of participation of multi-network hubs in their networks. To address the relationship between changes in network dynamics and ongoing cognition, we tested whether the dynamics of hub participation varied across individuals with respect to the contents of thought that they reported at the end of the resting-state experiment.

In the context of resting-state connectivity dynamics, recent reports about the importance of BOLD signal variability (Garrett et al., 2011, 2013) are of relevance. As Garrett et al. could show the signal variability is not only reduced in poorer performance (Garrett et al., 2013), but is also further diminished in older subjects (Garrett et al., 2011, 2013). Here, we also tested for a link between aging and a reduction of network dynamics.

\section{MATERIALS AND METHODS DATA AND SUBJECTS}

Data was acquired using a Siemens 3 Tesla Trio scanner and included resting-state functional magnetic resonance imaging (rs-fMRI) and a T1 anatomical scan. The rs-fMRI data were acquired over 900 volumes with 40 slices, a TR of $0.645 \mathrm{~s}$ and a resolution of $3 \mathrm{~mm}$ isotropic. The sequence (Xu et al., 2012) further comprised of the following parameters: $T E=30 \mathrm{~ms}$, flip angle of $60^{\circ}$ and a multiband factor of 4 . Subjects were instructed to keep their eyes open and fixate on a crosshair. T1 anatomical scans were obtained using an MPRAGE sequence with a resolution of $1 \mathrm{~mm}$ isotropic.

From the initial 231 subjects we excluded 7 due to imaging artifacts and 44 for having maximum motion of more than $3 \mathrm{~mm}$. To reduce potential micro motion artifacts we further removed 72 subjects with summed micro-movements (Van Dijk et al., 2012) above the group mean $(0.1152 \mathrm{~mm} /$ volume $)$. In addition, in the further analysis we still accounted for micro-movements as a covariate.

The resulting 108 subjects had a mean age of 37.71 years (std. 18.4 years) including 47 males and 61 females subjects. All data sets used in this study are part of the NKI Enhanced dataset (Nooner et al., 2012) and are made publicly available by the international neuroimaging data sharing initiative (Biswal et al., 2010). Institutional Review Board Approval was obtained at the Nathan Kline Institute and Montclair State University. Written informed consent was obtained for all study participants.

\section{PREPROCESSING}

The preprocessing of resting-state fMRI data was carried out using FSL (Jenkinson et al., 2012), AFNI (Cox, 1996), and
FreeSurfer (Dale et al., 1999). The steps included: (1) discarding the first four EPI volumes from each resting-state scan to allow for signal equilibration, (2) 3D motion correction, (3) slice time correction, (4) 4D mean-based intensity normalization, (5) removing linear trends, (6) regressing out 11 nuisance signals: six motion parameters and five top components from a principal components analysis of high variance signals (CompCor; Behzadi et al., 2007; Chai et al., 2012), and (7) band-pass temporal filtering $(0.01-0.1 \mathrm{~Hz})$. The output of these preprocessing steps is one $4 \mathrm{D}$ residual functional volume for each participant. In order to reduce partial volume effects no spatial smoothing was performed. We did not use global signal regression as the global signal is tightly coupled to the underlying neuronal signal (Schölvinck et al., 2010).

A non-linear transformation from $\mathrm{T} 1$ to a $3 \mathrm{~mm}$ isotropic $\mathrm{MNI}$ template (created from 152 subjects, provided with FSL) was calculated for individual T1 images using ANTs (Avants et al., 2011). This transformation was combined with the EPI to T1 transformation (bbregister Greve and Fischl, 2009) to warp the EPI volumes to standard MNI space.

The preprocessing pipelines used for this manuscript can be downloaded from https://github.com/alexschaefer83/ DynamicHubs and used together with BIPS (https:/github. com/INCF/BrainImagingPipelines) which is based on nipype (Gorgolewski et al., 2011).

\section{GRAPH CONSTRUCTION}

A graph is an abstract representation of a network. A graph G consists of a set of vertices $\mathrm{V}$ and a set of edges $\mathrm{E}$. An edge indicates the presence of a relationship between two vertices, which in case of this study is functional connectivity. We will also refer to edges as connections. To account for different strength of functional connectivity we use an edge weighted graph.

For the graph construction we parcellated the functional images into 200 cortical and subcortical regions. The parcellation is based on spatially constrained spectral clustering (Craddock et al., 2012) which aims to create spatially coherent regions of functional homogenous connectivity. The parcellation is publicly available (www.nitrc.org/projects/cluster_roi). In the proposed analysis we used the version derived from the best performing clustering in the publication by Craddock et al. (2012) (two-level clustering with the rt similarity metric). Each parcel is one unique vertex in our graph. To estimate the relationships between the vertices, the average signal within each parcel was extracted and its pairwise correlation with the signals (spatial averages over all voxels of a parcel) of all other parcels computed. The resulting correlation values were Fisher z-transformed in order to allow for an unbiased analysis in the further steps. We then averaged the $z$-values over all subjects. For the graph we assigned an edge (or connection) between two vertices (or parcels) if their respective correlation value belonged to the highest $10 \%$ (2000 edges) in the group average. Furthermore, we weighted the edge by the corresponding $z$-value. This technique shows good reliability (Schwarz and McGonigle, 2011) as it incorporates only strong connections with relatively high reliability (Patriat et al., 2013). Visualization of brain graphs was performed using braingl (Böttger et al., 2014) and conview (http://conview.googlecode.com). 


\section{CONNECTIVITY CLUSTERING}

An efficient way to cluster connections has been proposed by Ahn et al. (2010). We used an implementation of this idea by Kalinka and Tomancak (2011). In order to cluster connections one requires a measurement of their similarity. Ahn et al. (2010) proposed the Jaccard coefficient to estimate the similarity between connections $e_{i k}$ and $e_{j k}$ that share a vertex $k$ :

$$
S\left(e_{i k}, e_{j k}\right)=\frac{|n(i) \bigcap n(j)|}{|n(i) \bigcup n(j)|}
$$

where $n(i)$ and $n(j)$ is the first order neighborhood of vertex $i$ and $j$, respectively. An example for two connections with low similarity is illustrated in Figure 1A. The similarity is $S=1 / 3$ as the common neighborhood of vertices $i$ and $j$ is only vertex $k$. An example for two connections with high similarity $(S=1)$ is

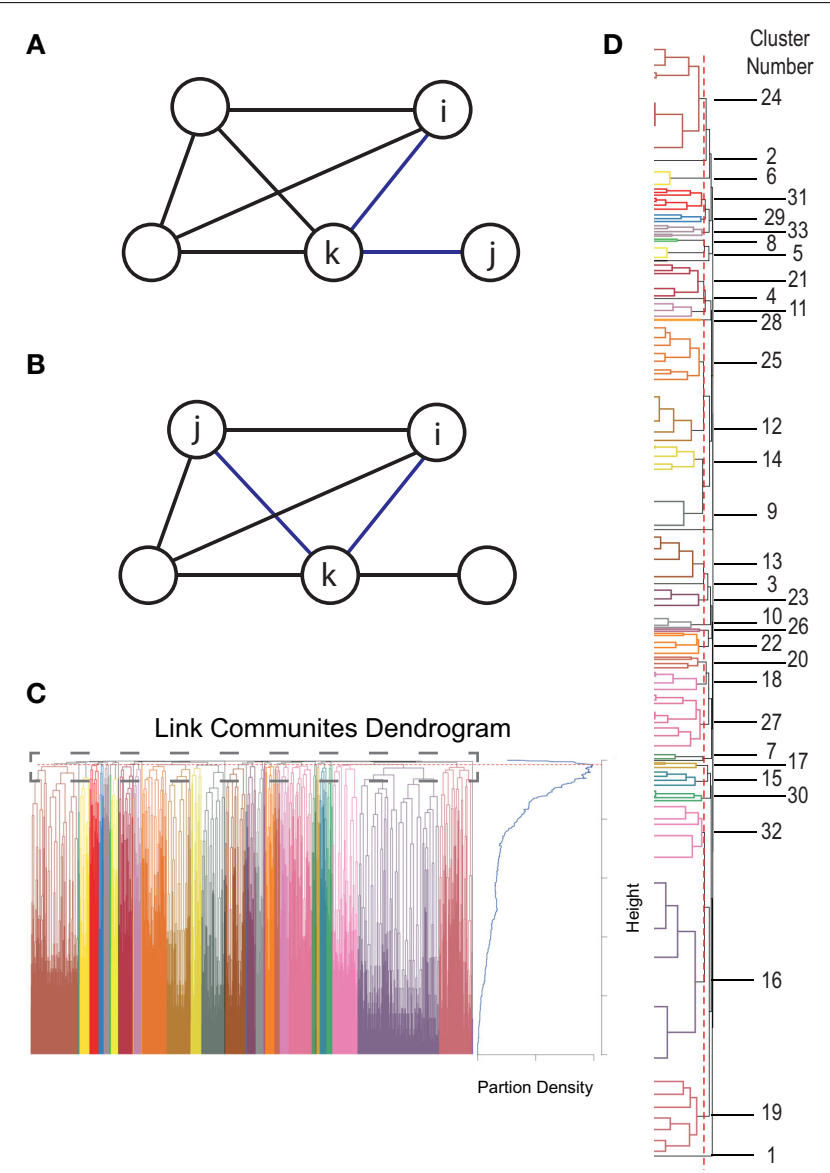

FIGURE 1 | Connectivity clustering approach. (A) Example for two connections with low similarity. The common neighborhood of vertices $i$ and $j$ is only $k$ therefore the similarity is $S=1 / 3$. (B) An example for two connections with high similarity. As vertices $i$ and $j$ have the same neighborhood the similarity is 1. (C) Dendrogram (left) based on calculated similarities together with a plot of estimated partition densities (right). Red dotted line indicates the cutoff of the dendrogram estimated by maximizing partition density. (D) Zoomed view of the top of the dendrogram from (C) together with network numbers from Figure 3 . illustrated in Figure 1B as $i$ and $j$ have the same neighborhood. To better account for the different strength of connections we use a weighted version called Tanimoto coefficient:

$$
S\left(e_{i k}, e_{j k}\right)=\frac{w_{i} w_{j}}{\left|w_{i}\right|^{2}+\left|w_{j}\right|^{2}-w_{i} w_{j}}
$$

where $\mathbf{w}_{i}$ is a vector describing the weights of the connections between vertex $i$ and the vertices in the first order neighborhood of $i$ and $j$. After calculating the pairwise Tanimoto coefficients between all links in the network, a hierarchical clustering is performed using McQuitty's similarity method (McQuitty, 1966). The optimal cutoff for the resulting dendrogram (tree diagram, Figure 1C) is determined by maximizing the partition density (Ahn et al., 2010). This is the density within the clusters, normalized for the maximum and minimum number of possible connections within each network. More explicitly, for a network with $M$ connections, $\left\{P_{1}, \ldots, P_{C}\right\}$ is a partition of the connections into $C$ subsets. A subset $P_{c}$ has $m_{c}=\left|P_{c}\right|$ connections and $n_{c}=\left|\bigcup_{e_{i j \in P_{c}}}(i, j)\right|$ vertices. Then Ahn et al. define the partition density of a subset $C$ :

$$
D_{c}=\frac{m_{c}-\left(n_{c}-1\right)}{\frac{n_{c}\left(n_{c}-1\right)}{2}-\left(n_{c}-1\right)}
$$

This is $m_{c}$ normalized by the minimum and maximum numbers of connections possible between $n_{c}$ vertices. The partion density $D$, is the average of $D_{c}$, weighted by the fraction of present connections:

$$
D=\frac{2}{M} \sum_{c} m_{c} \frac{m_{c}-\left(n_{c}-1\right)}{\left(n_{c}-2\right)\left(n_{c}-1\right)}
$$

The maximum partition density gives the optimal cutoff for the dendrogram which determines the number of connectivity clusters in our solution (Figure 1D).

\section{RELIABILITY OF CLUSTERING}

To evaluate the reliability of the new clustering method we use a split half test. In order to not compare clustering over different elements (connections) we used the same connections as in the full group for both clusterings (see Graph Construction). To evaluate reliability of the cluster results we divided our subjects into two groups, one of subjects with odd index and one of subjects with even index, and performed the clustering for both of them separately. As distance between the two found clustering solutions (even and odd index) we computed the so-called cophenetic correlation coefficient (Sokal and Rohlf, 1962). To compute confidence intervals for the results we performed a mantel statistic using 999 permutations (Mantel, 1967) which creates a permutation baseline. The two found clustering solutions correlated with $r=0.8954$ ( $p<0.001,95 \% \mathrm{CI}=$ $0.001185)$. The similarity of the results across subgroups implies a certain generalizability of our cluster results across a larger population. 


\section{WINDOWING OF TEMPORAL DYNAMICS}

To investigate the underlying dynamics of the resulting connectivity clusters instead of correlating signals over the whole scan session, a shifting time window is used:

$$
r_{t}=\operatorname{corr}\left(x\left(t \cdot w_{. .}(t+1) \cdot w-1\right), y\left(t \cdot w_{. .}(t+1) \cdot w-1\right)\right)
$$

where $t$ is the timepoint with $t=1 . .11, w$ is the window with $w=77$ (or 49, $7 \mathrm{~s}$ ) and $x, y$ are the time series of two (out of 200) parcels. All estimated $r$-values are Fisher's $z$-transformed, resulting in $11 z$-values per connection and subject. Each of these 11 values is adjusted to a within subject baseline by subtracting from the estimated $z$-value for the complete scan time. These normalized values reflect, for each subject, the temporal change of the connection strength over the 11 time intervals.

\section{ANALYSIS OF TEMPORAL DYNAMICS}

To investigate if the connections within a connectivity cluster change, over time, more similarly than over connectivity clusters we measured the variance of connectivity change within (var1, var2 in Figure 2B) and without clusters (var3 in Figure 2B). Timepoints were not averaged over subjects as subjects are likely in a different state of dynamic functional connectivity and therefore not comparable. We tested whether the within cluster variance was significantly smaller to the variance over clusters using a Wilcoxon test.

To analyze if regions in multiple networks Figure 2A show a dynamically varying degree of membership between its belonging networks we used the following strategy. To test if a region changes the degree of membership between its networks, we estimated the average strength of connections assigned to one network and compared it to the average strength of connections belonging to another network using a dynamic windowing (50 s) approach. These "variation" events are illustrated as State $\mathrm{B}$ and State D scenario in Figure 2C and can be well-captured by the mean squared error (MSE) between the average correlation strengths. To test the significance of our results we used permutation testing based on random clustering. In this clustering we chose randomly connections from the same hub regions without caring about assigned cluster values. The number of connections was equal to the original clusters. We created 10,000 times two corresponding random clusters and computed the MSE between them. The results are plotted in Figure 5C.

For the whole brain analysis we aimed at ensuring that networks tested included enough connections for a stable signal. Therefore, we chose only regions where the second largest network included at least two connections (151 of 200 regions, Figure 4C). The MSE was then averaged over all of pairs of networks which shared participation of the particular hub.

\section{EXPERIMENT: SELF-GENERATED THOUGHTS}

To assess thoughts and feelings during the scan the subjects were interviewed directly after MRI session using the New York Cognition Questionnaire (NYC-Q). The beginning of the interview was approximately $45 \mathrm{~min}$ after the resting state scan that we analyzed. The NYC-Q is a self-report tool consisting of two sections, the first containing questions about the content of thought (23 questions), the second containing questions about the form that these thoughts take (8 questions). In each question subjects were asked to indicate how well a statement described their thoughts on a scale from 1 ("Completely did not describe my thoughts") to 9 ("Completely did describe my thoughts").
A

Region in Two Networks

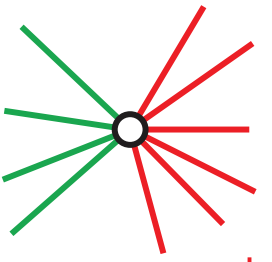

red network

green network

C

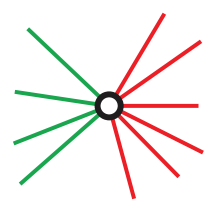

State A

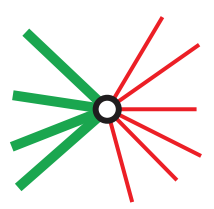

State B
B

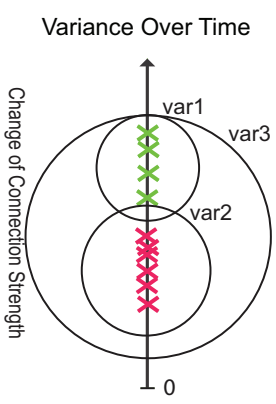

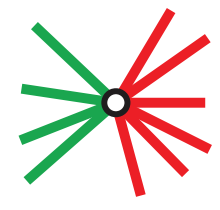

State C

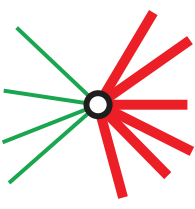

State D line thickness indicates connection strength

green cluster, while var3 gives the variance over all connections. (C) Four possible scenarios of changing connectivity: State A: reduction of both clusters, State B: reduction of red cluster and increase of green cluster, State $C$ : increase of both clusters, and State D: reduction of green cluster and increase of red cluster. 
Therefore, a high score on a component relates to the subjective experience of mind-wandering which also implies that one strong thought yield a similar score as multiple seemingly insignificant thoughts. We used the data and code available online (https:// github.com/NeuroanatomyAndConnectivity/NYC-Q) provided and described in greater detail by Gorgolewski et al. (in press). The 23 answers about the content of thought collected from 166 subjects were factorized into five categories. The factorization was performed using principal axis factor analysis together with an oblimin rotation (Revelle, 2011) to increase interpretability. The number of factors was estimated using Parallel Analysis (Horn, 1965). Individual-level scores were computed applying the method by Ten Berge et al. (1999). We used the names and interpretation of these categories as described in the original study, namely thoughts about the past (Past), the future (Future), positive thoughts (Positive), negative thoughts (Negative), or thoughts about relationships (Social Cognition). The eight questions about the form of thought were factorized into three factors as described above. The factors were named as in the original study: in form of words (Words), in form of images (Images), and specificity of words (Vague). As the factorization employed does not enforce orthogonality of the components, we performed for each component a partial correlation analysis with the respective other seven components as covariates. A more detailed description about NYC-Q and the factorization employed can be found in the study by Gorgolewski et al. (in press).

\section{RESULTS HUB REGIONS BELONG TO MULTIPLE NETWORKS}

Using the edge clustering approach described above (Ahn et al., 2010) we found 33 networks (Figure 3), several of which are well-characterized networks typically observed in fMRI resting-state experiments. The clusters can be downloaded from https://github.com/alexschaefer83/DynamicHubs or interactively viewed online http://openscience.cbs.mpg.de/schaefer. The number of connections and regions in each network can be found in Table 1. We found that the majority of regions (174 of 200) participated in more than one network. An overview of the amount of multi-network participation is given in Figure 4B. In contrast Figure 4A gives the degree of connectivity of these regions. The relation of the two measures connectivity and multi-network participation is given in Figure 4D.

\section{HUB REGIONS VARY DEGREE OF MEMBERSHIP BETWEEN NETWORKS}

As an example Figure 5 presents the results for the analysis on the anterior cingulate cortex (ACC). While an overview for whole brain results is given in Figure 4C, we chose the ACC as a representative area to illustrate the typical results for a single area. We found the ACC to participate in two spatially separated networks: a temporal network and a frontal-parietal network (Figure 5A).

To test if connectivity changes occur more within clusters than between clusters we estimated the variance of connectivity change across timepoints and subjects. We found the variance of change within the red cluster $(p=0.0181)$ and within the blue cluster $\left(p=1.156 * 10^{-23}\right)$ to be smaller than the variance of connectivity change over both clusters.
To test if a region changes the degree of membership between its networks, we estimated the average strength of ACC connections assigned to one network and compared it to the average strength of ACC connections belonging to another network. As shown in Figure 5B, we found changes in the degree of membership, which we quantified by the mean squared difference in participation. We used permutation tests to show that these changes between networks were statistically significant, see Figure 5C. We found there were significantly stronger changes in the degree of participation between specific clusters than would be expected between randomly selected clusters (Figure 5C).

A whole-brain overview of the amount of variation in network participation can be seen in Figure 4C. We also investigated if there is a relation between dynamic (Figure 4C) and connectivityhubness (Figure 4A) or network-hubness (Figure 4B). We found a positive correlation (Spearman $r=0.44, p=2 * 10^{\wedge}-8, n=$ $151)$ between variation of network participation and the degree of connectivity (Figure 4E) while we accounted for the number of networks. Further, we also found a weaker correlation (Spearman $r=0.16, p=0.049, n=151$ ) between the dynamics of a region and the amount of networks the region participates in (Figure 4F) while accounting for the number of connections.

\section{ASSOCIATION BETWEEN VARIATION OF NETWORK PARTICIPATION AND SELF-GENERATED THOUGHTS}

We found a reduction of whole brain-averaged variation of network participation in subjects which reported to have more positive thoughts during the scan [Figure 6C, Spearman $r=-0.47, p=0.0001$ (corrected for 8 comparisons), $n=78$ ]. An overview of whole brain participation variation is also given in Figure 4D. We further investigated if the variation of network participation of a specific region correlates with a domain of self-generated thoughts. We found an increased variation of network participation of the left caudate in subjects which reported to have more thoughts about the past during the scan \{Figure 7, Spearman $r=0.47, p=0.02$ [corrected for 1600 comparisons ( 8 categories $* 200$ regions) $], n=78\}$.

\section{DECREASE OF DYNAMICS IN AGE}

As the variability of the fMRI signal has been found to decrease in aging (Garrett et al., 2011, 2013), we tested whether similar effects can be found for the dynamic of network participation of brain regions between networks. Critically, we found a significant age effect on connectivity changes across the whole brain (spearman $r=-0.24, p=0.011, n=106$, Figure 6A). While we used micro-movements as a covariate we also tested for a relationship between network dynamics and micro-movements. However, we did not find a relation between whole brain dynamics and micromovements ( $r=0.03, p=0.747, n=106$, Figure 6B). We found no significant correlation between age and positive self-generated thoughts (SGT) corrected for micro-movements $(r=0.111, p=$ $0.338, n=78$, Figure 6D). However, when we added positive SGT as a covariate the negative correlation between age and participation remained similar (spearman $r=-0.24, p=0.033$, $n=78$, Figure $6 \mathrm{E}$ ).

The impact of different initial graph thresholds onto the three main results of this paper are shown in Table 2. In 


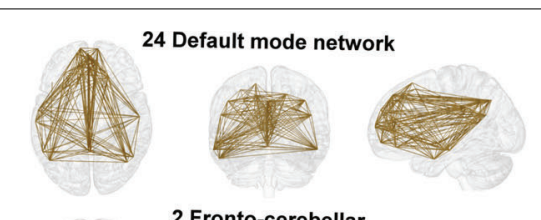

2 Fronto-cerebellar
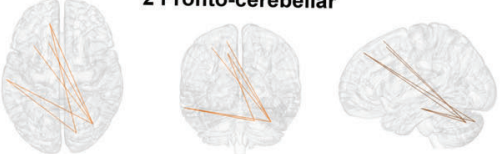

6 Fronto-parietal
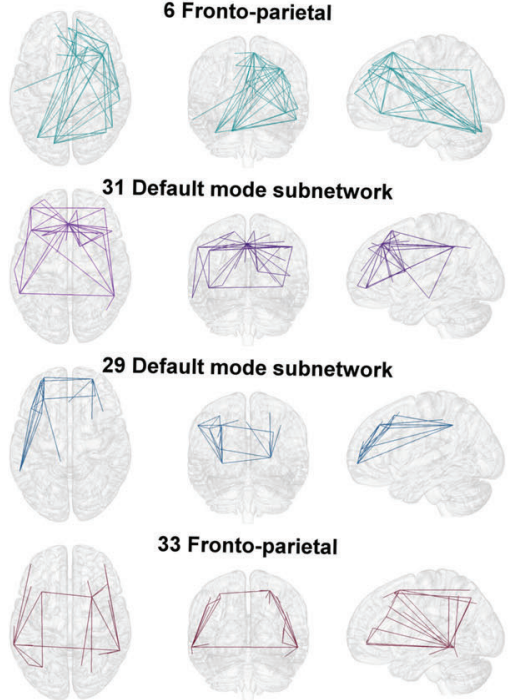

33 Fronto-parietal
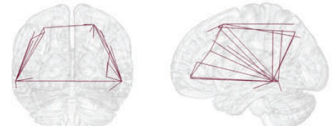

8 Fronto-parietal
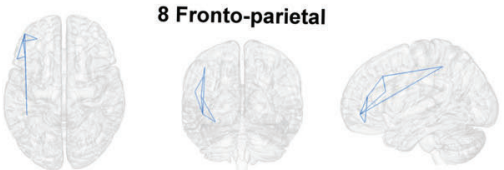

5 Fronto-parietal
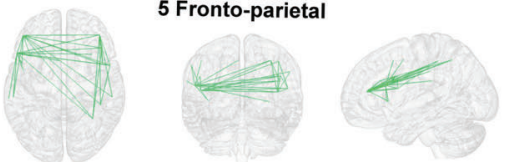

21 Subcortical-cerbellar

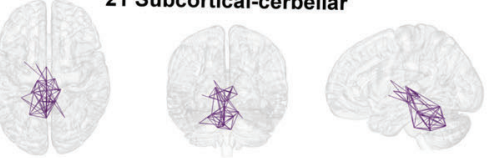

4 Subcortical-cerbellar
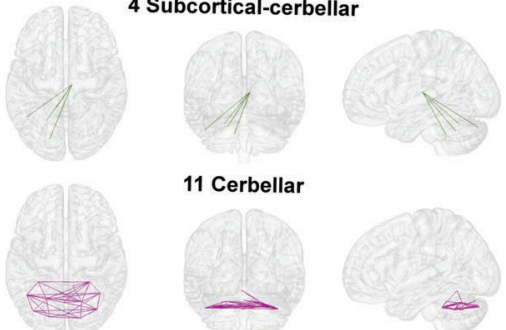

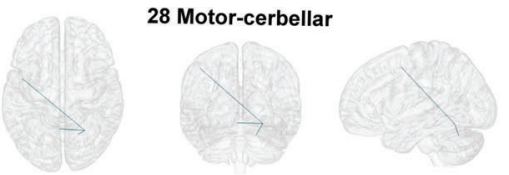

25 Higher-order visual
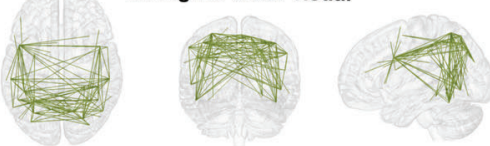

12 Higher-order visual
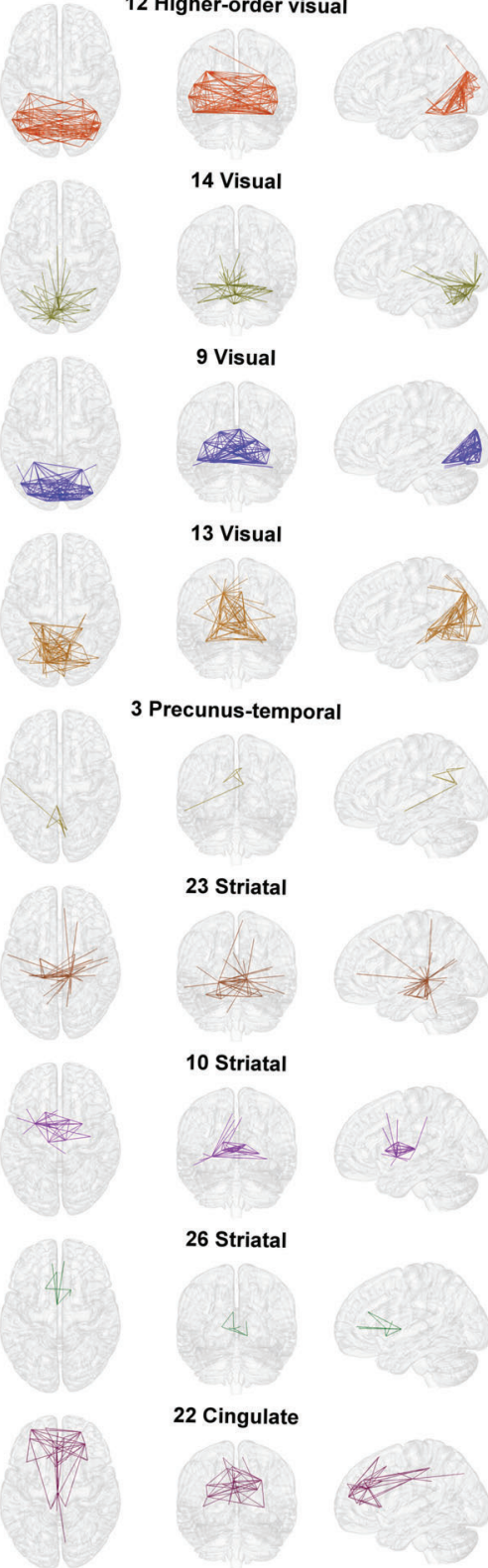

9 Visual

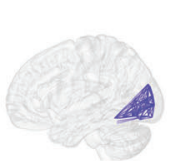

13 Visual
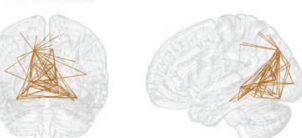

3 Precunus-temporal
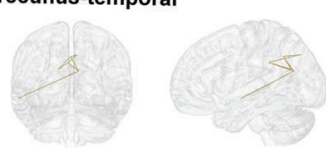

23 Striatal
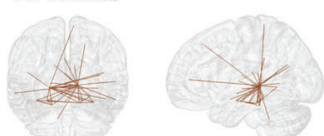

10 Striatal
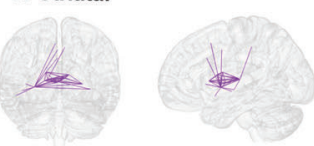

26 Striatal

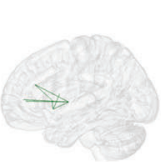

22 Cingulate
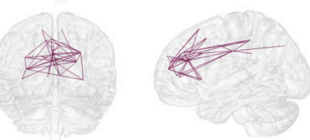
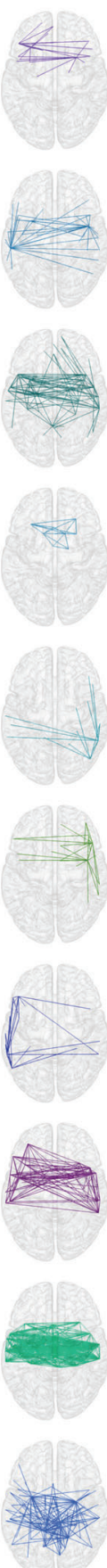

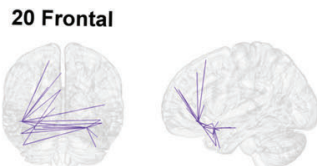

18 Temporal
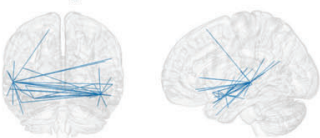

27 Temporal-frontal

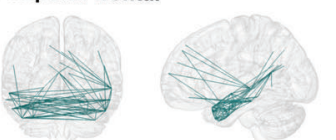

7 Striatal

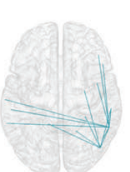

9 Somatosensory
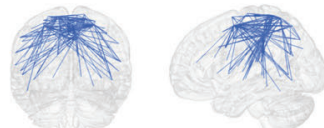

1 Precuneus-tempora

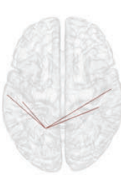

17 Lateral-occipital

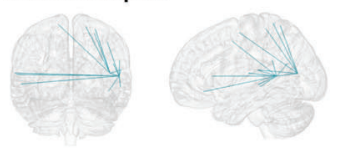

15 Premotor
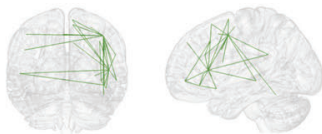

30 Language
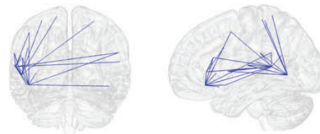

32 Premotor
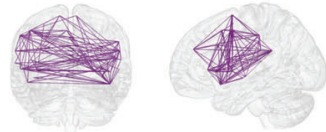

16 Sensorimotor
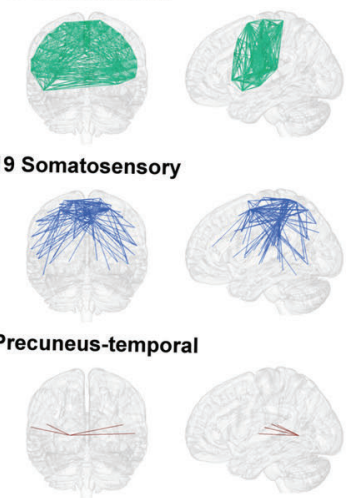

FIGURE 3 | Connectivity networks. All 33 connectivity networks found in the hierarchical cluster analysis of time and group averaged connectivity. Networks can also be inspected interactively and in three dimensions online (http://openscience.cbs.mpg.de/schaefer).

this manuscript we used a threshold of $10 \%$ or 2000 connections. All results remained significant for thresholds from $7.5 \%$ (1500 connections) to $15 \%$ (3000 connections). For a threshold of $17.5 \%$ (3500 connections) the caudate finding became insignificant. The clustering of connectivity for sparsity thresholds of $20 \%$ and higher resulted in a single network, whereas for sparsity threshold of 5\% and lower the left caudate was part of a single network. In this context no effect sizes were calculated. 
Table 1 | Descriptive information of connectivity networks (Figure 3).

\begin{tabular}{|c|c|c|}
\hline Network & Vertices (regions) & Edges (connections) \\
\hline 1 & 5 & 8 \\
\hline 2 & 6 & 6 \\
\hline 3 & 6 & 8 \\
\hline 4 & 5 & 4 \\
\hline 5 & 16 & 33 \\
\hline 6 & 18 & 46 \\
\hline 7 & 8 & 18 \\
\hline 8 & 6 & 8 \\
\hline 9 & 19 & 105 \\
\hline 10 & 16 & 36 \\
\hline 11 & 14 & 35 \\
\hline 12 & 24 & 109 \\
\hline 13 & 31 & 92 \\
\hline 14 & 34 & 49 \\
\hline 15 & 21 & 30 \\
\hline 16 & 33 & 369 \\
\hline 17 & 16 & 16 \\
\hline 18 & 32 & 41 \\
\hline 19 & 59 & 147 \\
\hline 20 & 19 & 20 \\
\hline 21 & 20 & 64 \\
\hline 22 & 21 & 42 \\
\hline 23 & 28 & 41 \\
\hline 24 & 26 & 217 \\
\hline 25 & 38 & 111 \\
\hline 26 & 7 & 7 \\
\hline 27 & 34 & 106 \\
\hline 28 & 4 & 3 \\
\hline 29 & 14 & 23 \\
\hline 30 & 19 & 26 \\
\hline 31 & 25 & 41 \\
\hline 32 & 31 & 114 \\
\hline 33 & 20 & 25 \\
\hline Average & 20.5 & 60.6 \\
\hline
\end{tabular}

Connectivity networks together with their respective number of vertices (regions) and number of edges (connections).

\section{DISCUSSION}

We have shown that multi-network hubs vary their degree of participation into different networks over time. In addition, we found that these network dynamics were inversely related to age and to positive self-generated thoughts reported by subjects. These results demonstrate a novel analytic approach which enables a testable framework for quantifying dynamic network interaction across regions on an individual-level.

To facilitate this analysis we used an algorithm to cluster connectivity itself rather than brain regions based on their connectivity profiles (Power et al., 2011). This approach offers the advantage to maintain whole brain information in connectivity space rather than reducing it to the regional space. This is important as we are interested in functional brain networks which includes connections and regions rather than only regions (Damoiseaux et al., 2006; Power et al., 2011). This domain shift also includes the conceptual advantage that brain regions can participate in multiple networks. While the later advantage is shared by other network decomposition methods which allow networks to overlap like independent component analysis (ICA, Calhoun et al., 2001; Beckmann et al., 2005), non-negative matrix factorization (NMF, Lee et al., 2011), and Latent Dirichlet Allocation (LDA, Yeo et al., 2014) it is still conceptually different as these networks exist in the spatial location but not in the connectivity space.

One of the limitations of connectivity clustering is that an initial arbitrary threshold onto the connectivity has to be applied. While we found that the effect sizes of our main results are comparable at a range of thresholds 7.5 to $15 \%$ (Table 2) we also found that for a threshold of the $17.5 \%$ the relation between caudate switching and past self-generated thoughts was insignificant. One reason for this might be the higher connectedness of the left caudate at this threshold which makes the left caudate a part of 10 networks, in contrast to two networks at $10 \%$ sparsity. For even higher thresholds the network clustering resulted in a single network. In this context a threshold between 7.5 and 15\% seems recommendable for similar future analyses.

While brain regions in our framework are allowed to participate in multiple networks we tested the hypothesis that this participation is not necessarily static over time. To illustrate this using the example of the ACC, Figure 5 shows a region in the ACC which takes part in two networks (networks 24 and 27). The [blue] network is spatially similar to the medial temporal lobe subsystem of the default mode networks (DMN), as described by Andrews-Hanna et al. (2010) and the [red] network is spatially similar to the dorsal medial prefrontal cortex subnetwork of the DMN (Andrews-Hanna et al., 2010). However, we found that the participation of the ACC region in these two subnetworks is not static over time (Figure 5B). This offers further interpretation of how these two subnetworks may interact in the DMN: the affiliation of this portion of ACC varies between them over time. Additionally, the variation network participation in single brain regions may in part account for the diversity of functions often associated with hub regions (Cole et al., 2013) such as the ACC (Devinsky et al., 1995).

While a recent primate study has shown dynamic of functional connectivity even in the absence of consciousness (Hutchison et al., 2013b), more recent human studies show relations of dynamic functional connectivity and physiology (Chang et al., 2013) as well as task performance (Thompson et al., 2013). Here we expand this picture by showing a relationship between dynamic functional connectivity and ongoing thought processes.

We found that variation in network participation was correlated with self-generated thought content across individuals; specifically it was related to thoughts about the past and those with a positive tone. Neurobiological studies suggest that there exist at least two different memory systems, one more cognitive system which relies upon medial temporal lobe and hippocampus areas and a stimulus-response system which banks in the basal ganglia (Poldrack and Packard, 2003). The default-mode network is a cortical network that connects middle temporal with the posterior cingulate and prefrontal areas, the subcortical network associated with the left caudate connects subcortical 
A

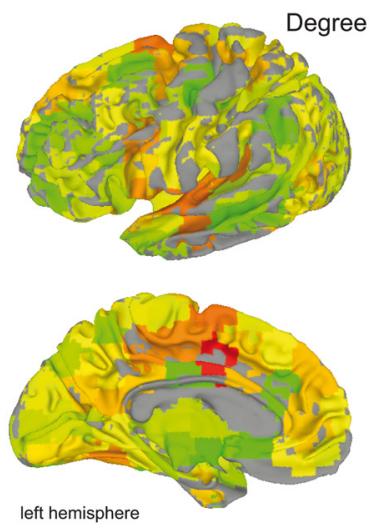

0
Degree of Connections
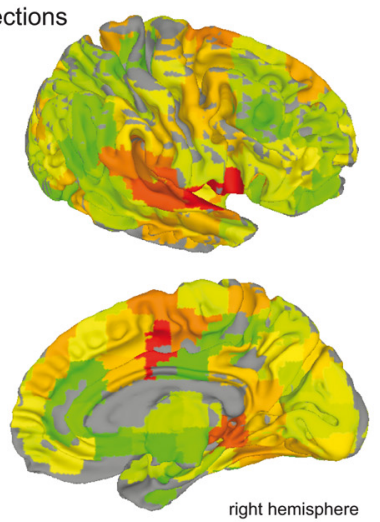

right hemisphere

B

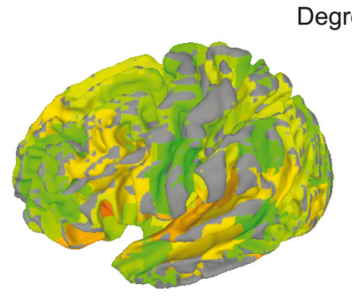

Degree of Networks
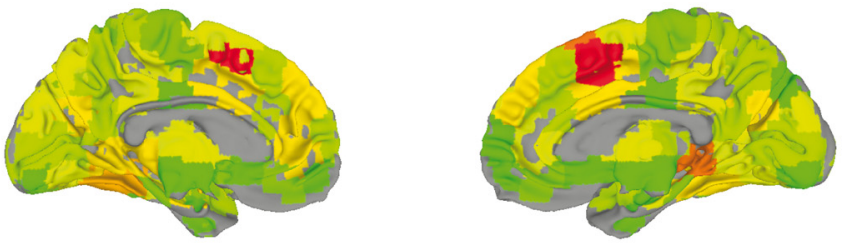

0

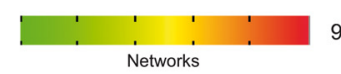

C

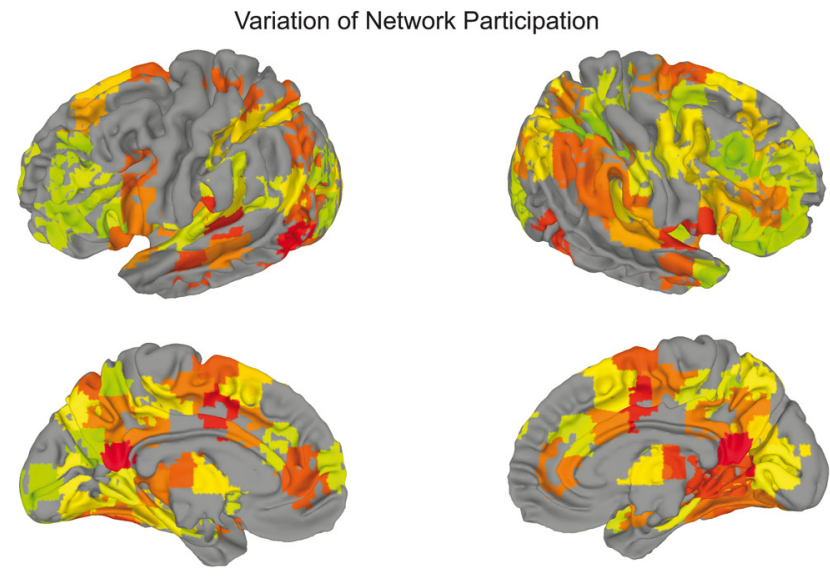

0

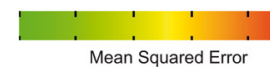

2.7

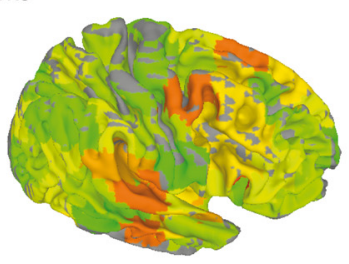

E

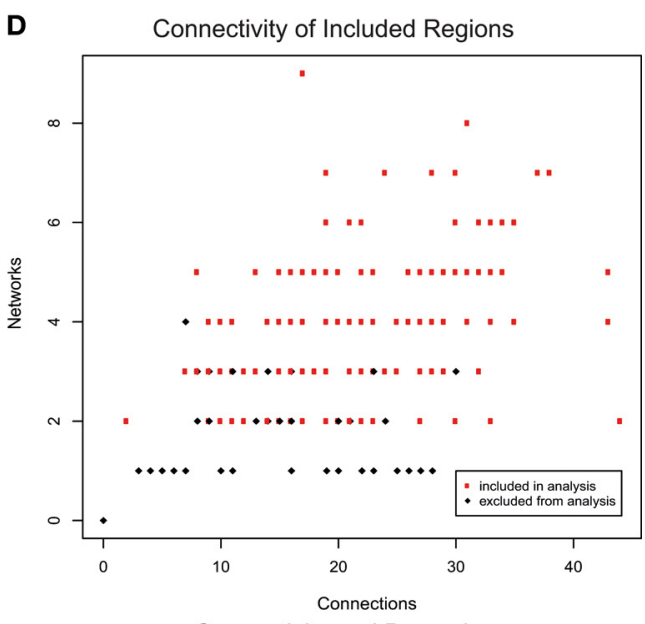

Connectivity and Dynamics

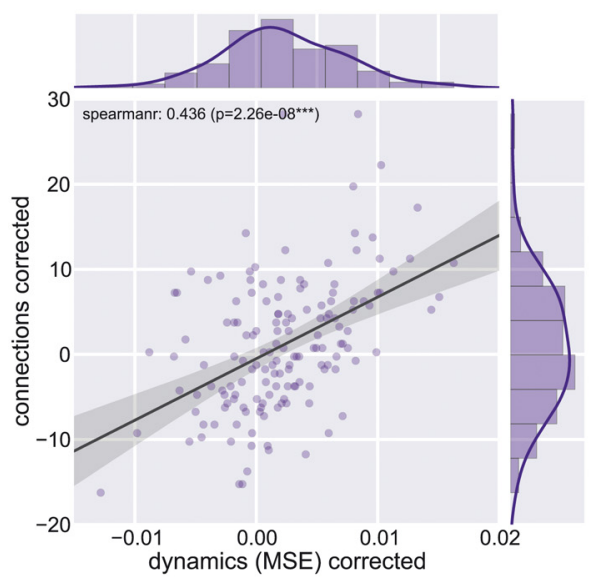

F Networks and Dynamics

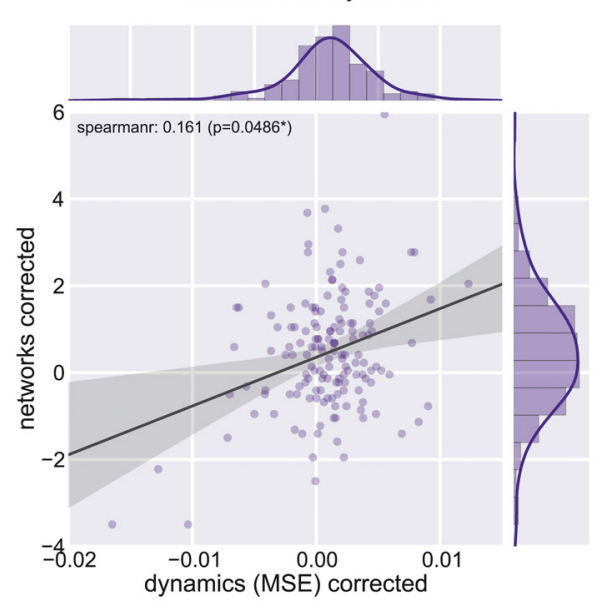

FIGURE 4 | Whole brain regional overview. (A) Brain areas colored by their degree of connectivity. Areas with more connections are displayed in red, whereas regions with fewer connections are in green. (B) Brain areas colored by the number of networks they are part of. Regions participating in a higher number of networks are displayed in red. Regions which are part of fewer networks are depicted in green. (C) Brain areas colored by their variation of network participation. Areas with higher variation are colored in red, whereas regions with lower variation are shown in yellow and green. (D) Relation

between number of connections and number of networks each brain region is part of. In the further analysis we only included regions which are part of at least two networks and for which the second largest belonging network consisted of at least two connections. These regions are colored in red. (E) Relation between number of connections and variation of network participation measured across brain areas included in analysis. (F) Relation between number of networks and variation of network participation measured across brain areas included in analysis. 

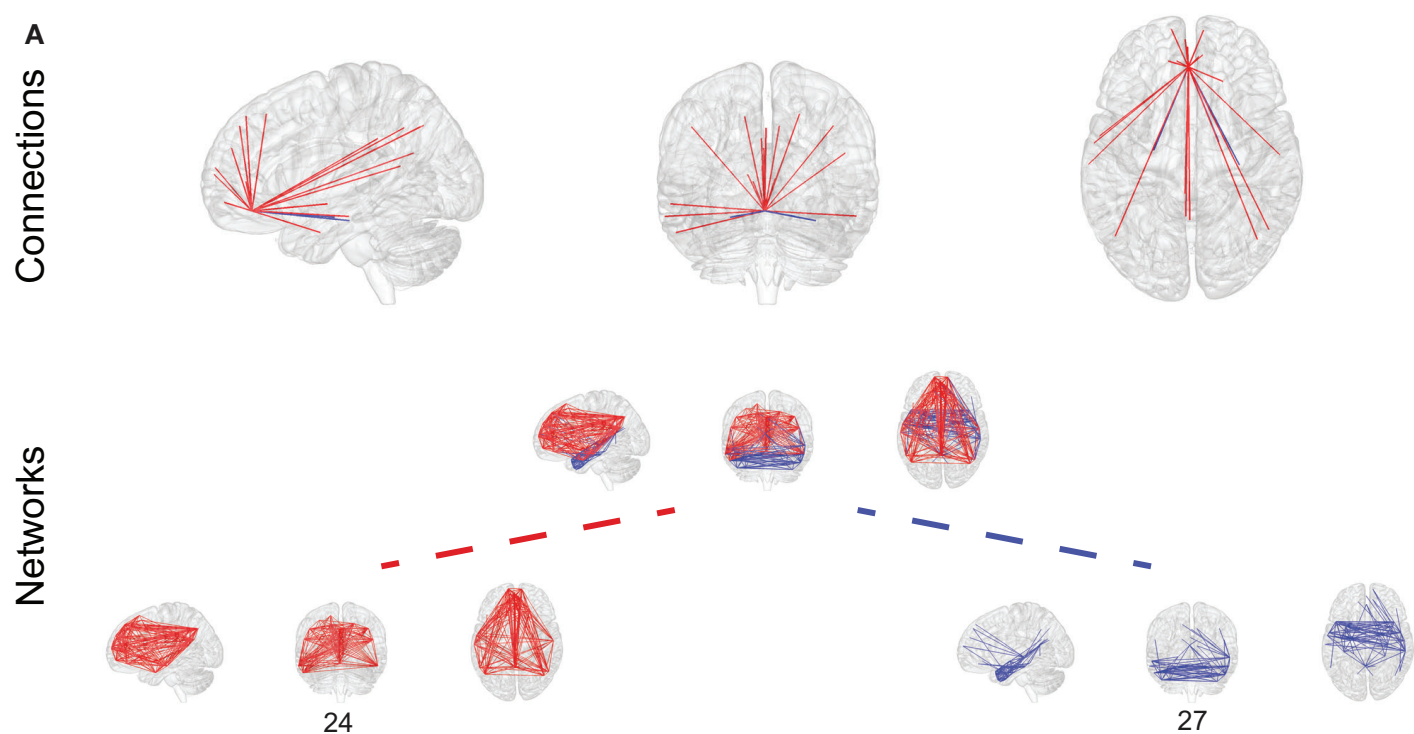

B

C
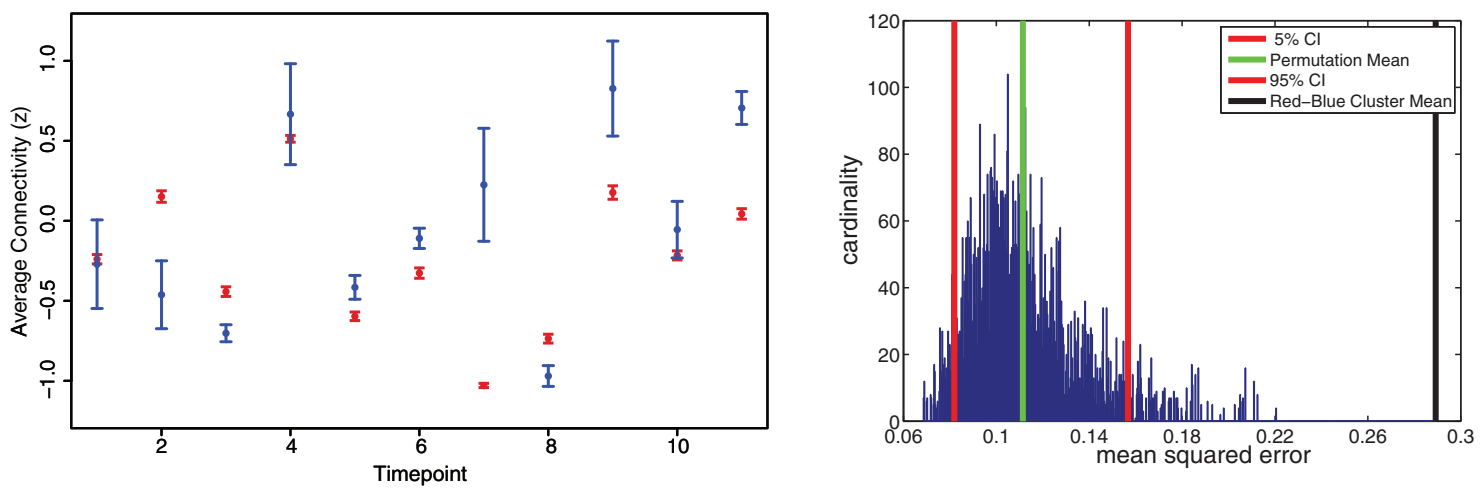

FIGURE 5 | Representative region for variation of network participation. (A) Anterior cingulate cortex (ACC) participating in two networks (networks 24 and 27). (B) Example plot of ACC participation in the two networks in a $50 \mathrm{~s}$ interval. Timepoint 2 and 7 are good examples of a variation in network participation (State B or State D, Figure 1C). (C) Permutation test shows that variation behavior measured by mean squared error is more pronounced in the found clustering than in a random clustering. regions such as thalamus, putamen, and caudate. Here we found that the left caudate is a hub involved in varying its degree of participation between these two networks and its specific behavior was correlated with self-generated thoughts about past events.

Self-generated thoughts about the past are known correlates of unhappiness (Smallwood and O'Connor, 2011) and may mark the temporal precursor of negative mood (Ruby et al., 2013). Self-generated thoughts of the past are also accompanied by greater disengagement, or decoupling, from external processes, as indexed by worse task performance (Smallwood et al., 2007, 2009). Altogether these studies illustrate that retrospective self-generated experiences may at times be both intrusive and unpleasant. The heightened variation in network participation of the caudate nucleus with increasing retrospective focus could therefore reflect the greater competition that accompanies especially repetitive or intrusive self-generated thoughts. Broadly consistent with this account we found that positive thoughts were associated with less variation in network participation at a whole brain level. In contrast to retrospective thoughts, pleasant experiences were associated with more consistent network dynamics, possibly reflecting the relatively calm form that positive experiences may take.

Variability of the fMRI signal, an univariate measure of brain dynamics, has been found to play an important role in behavioral performance (McIntosh et al., 2010; Garrett et al., 2013). One crucial observation is its reduction in older individuals or those who performing poorly in a variation of cognitive tasks (Garrett et al., 2013). While the biological reason for the loss of variability might arise from a dysregulation of dopamine and glutamate (Hong and Rebec, 2012), the behavioral implications might be explained by computational models (Deco et al., 2011). These models suggest that variability of brain signal is important for exploring the repertoire of possible brain states (Deco et al., 2011), while lower variability will raise the potential for remaining in a single state-resulting in inflexible 


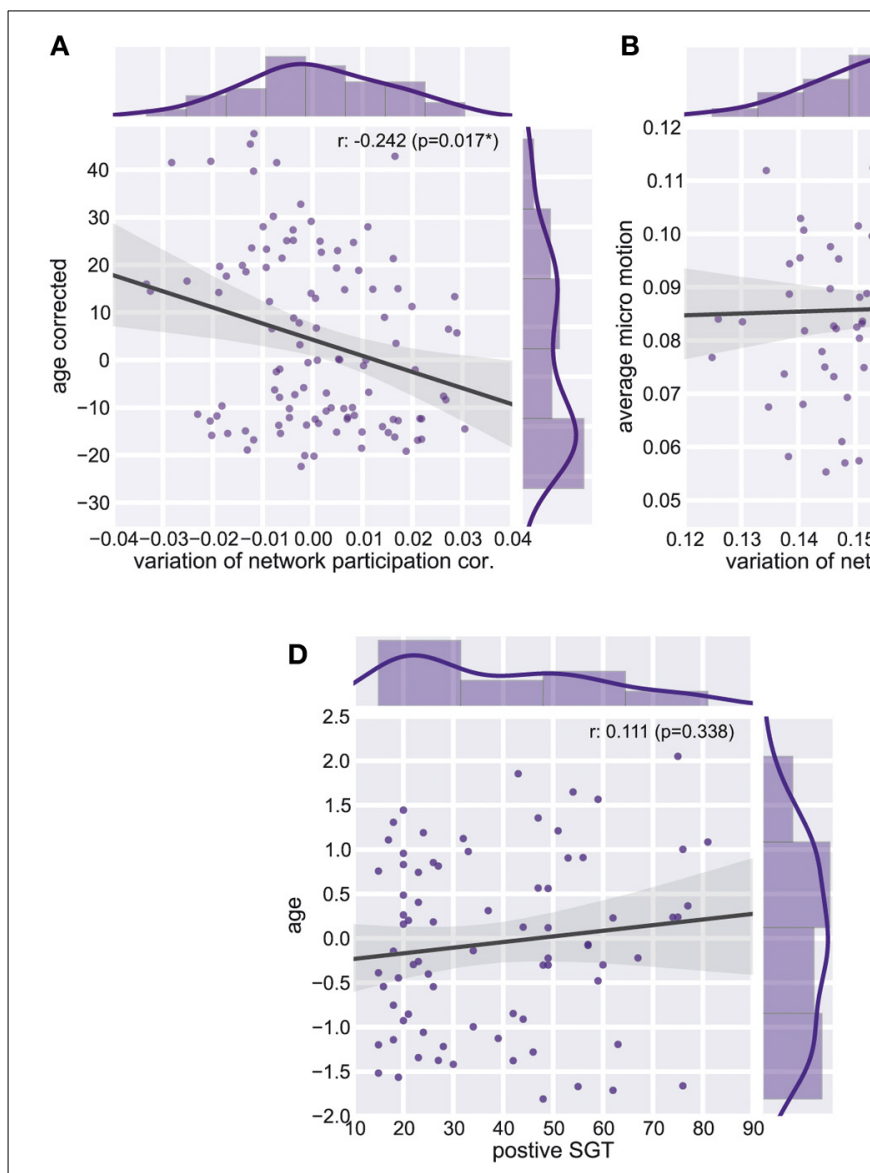

FIGURE 6 | Whole Brain Variation of Participation. (A) Partial correlation between average variation of participation and age corrected for micro-movements. (B) Correlation between average variation and average micro-motion. (C) Partial correlation between average variation of participation and positive self-generated thoughts (SGT) corrected for

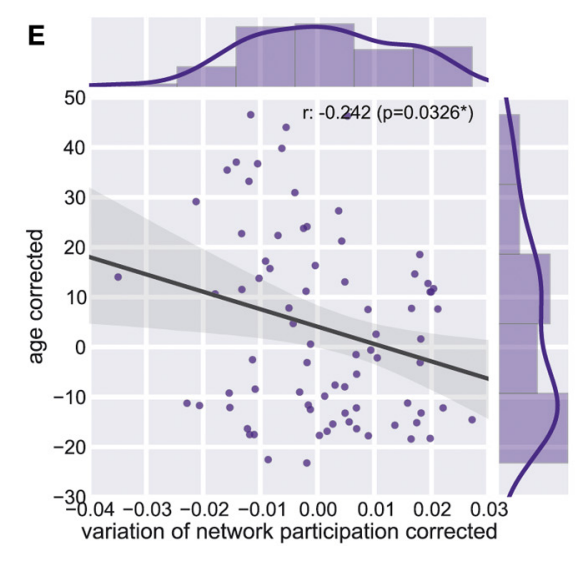

micro-movements, age, gender, and the respective other four factors of SGT. (D) Correlation between age and positive self-generated thoughts (SGT) corrected for micro-movements. (E) Partial correlation between average variation of participation and age corrected for positive self-generated thoughts (SGT), micro-movements. behavior. Here we have shown a decline of variation in network participation during aging. Aging is therefore not only associated with decreased signal variability but also reduced interplay between networks, suggesting that dynamic network participation may underlie behavioral flexibility. However, the interaction of dynamics and age was not linear, indicating a more complex relation between dynamics and age which might be investigated in future studies.

Interactions of networks have been studied to date with respect to their average signal. The anticorrelation between the average signal of the DMN and the average signal of the task positive network has been found to be predictive of individual behavior during task and rest (Kelly et al., 2008). Recently it has been shown that this relationship changes during the task based on the current performance (Thompson et al., 2013). A relationship between this anticorrelation and variation in network participation is possible, but not straightforward. As anticorrelation is based on the average signals of all vertices in respective networks the variation in network participation describes the varying integration of single regions into the networks.
In the analysis of dynamic network participation we only included regions which belonged to two or more networks and where the second largest consisted of at least two connections (Figure 4D). While this property is fulfilled by all highly connected regions (Figure 4D), there were also few sparsely connected areas included into the analysis. In this context we focused stronger on multi-network hubs then their pure connectedness, while we also found a correspondence between these two properties (Figure 4D).

While we hypothesized that dynamic network interaction takes place in highly connected areas we also found that the extent of these dynamics is related to the extent of the hubness (Figure 4E). This evidence further supports our hypothesis that hub areas might serve as relay stations which enable information integration. However, the effect of multi-network hubness was much weaker (Figure 4F) and more connections could just improve the detectability of the underlying dynamic process.

The description of dynamic organization in resting-state connectivity (Majeed et al., 2009; Britz et al., 2010; Chang and Glover, 2010; Musso et al., 2010; Handwerker et al., 2012; Smith et al., 


\section{A}
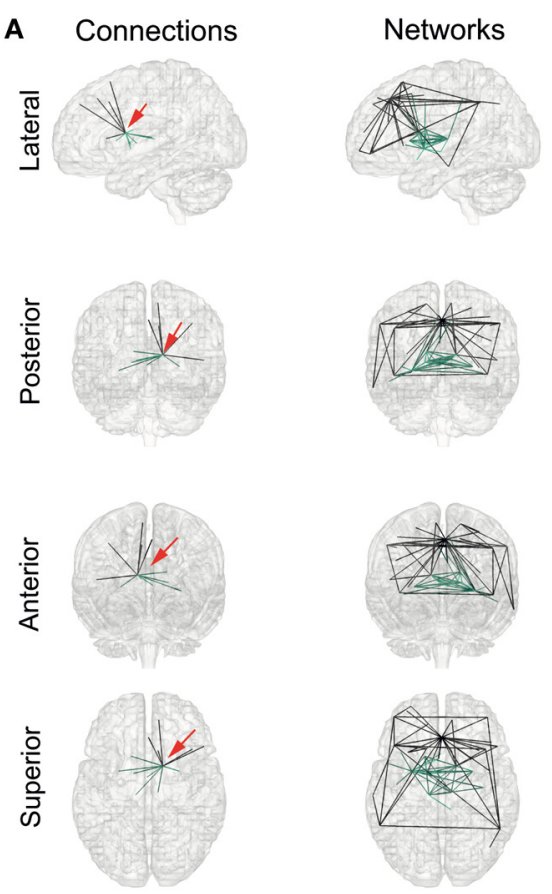

B

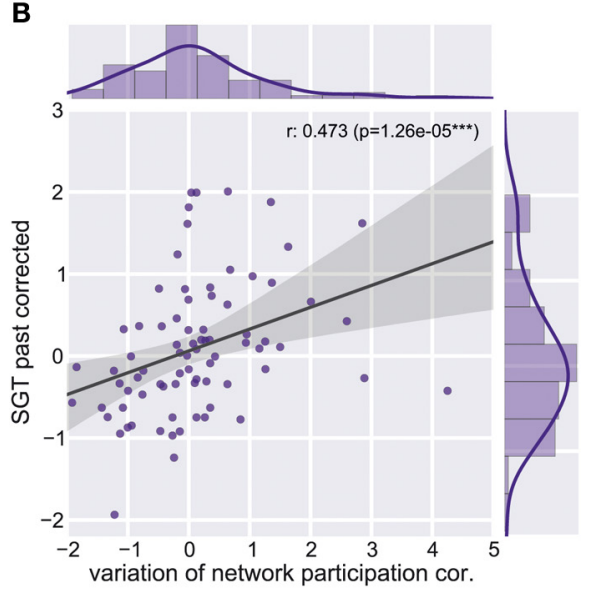

FIGURE 7 | Left caudate region and self-generated thoughts about past events. (A) Left caudate region is part of two connectivity networks, a sub cortical network (network 10) and a subnetwork of the default mode network (network 31). Red arrow indicates the position of the left caudate region. (B) Increased correlation between variation of network participation of the left caudate region and self-generated thoughts about past events.

2012) raised concerns about the potential artifactual origin of these BOLD synchrony fluctuations. Studies using EEG-fMRI data, however, have established a neuronal origin of dynamic resting-state connectivity (Britz et al., 2010; Musso et al., 2010; Tagliazucchi et al., 2012). Another recent functional connectivity study in anesthetized macaques demonstrated dynamic functional connectivity in the absence of any motion (Hutchison et al., 2013b). In order to avoid potential confounds in our study, we selected datasets with minimal motion and tested for remaining influences. We did not find that subject motion accounted for the increased variation in network participation (Figure 6B).
Table 2 | Impact of different initial graph thresholds for the main results of the manuscript.

\begin{tabular}{lccc}
\hline Threshold & $\begin{array}{c}\text { Whole brain VNP/ } \\
\text { positive SGT }(\boldsymbol{r})\end{array}$ & $\begin{array}{c}\text { whole brain } \\
\text { VNP/age }(\boldsymbol{r})\end{array}$ & $\begin{array}{c}\text { Caudate VNP/ } \\
\text { past SGT }(\boldsymbol{r})\end{array}$ \\
\hline $17.5 \%$ (3500 edges) & -0.46 & -0.21 & 0.14 \\
$15 \%$ (3000 edges) & -0.43 & -0.26 & 0.41 \\
$12.5 \%$ (2500 edges) & -0.49 & -0.20 & 0.38 \\
$10 \%$ (2000 edges) & -0.47 & -0.24 & 0.47 \\
$7.5 \%$ (1500 edges) & -0.46 & -0.21 & 0.40
\end{tabular}

The threshold of $10 \%$ (2000 edges) was used in this study. Main results are partial correlation between whole brain averaged Variation of Network Participation (VNP) and positive Self-Generated Thoughts (SGT), whole brain averaged VNP and age, VNP of left caudate and SGT about the past.

A limitation of the current study is the fixed window length of $50 \mathrm{~s}$ which we chose based on the size of our bandpass filter ( $10 \mathrm{~s}$ to $100 \mathrm{~s}$ ). This length might not always coincide with the dynamic of ongoing cognitive processes. To give evidence for the robustness of our findings we reanalyzed the data with window lengths of $65 \mathrm{~s}$ (100 volumes). All our main results remained significant. However, a data-driven approach to detect temporal change points as described recently by Cribben et al. (2012) gives an adaptive window length which could further enhance sensitivity to dynamic processes. A potentially confounding influence is the variation of node sizes which could affect the extracted time series differently by noise. While the parcellation used in this study aimed to reduce the variation of node sizes (Craddock et al., 2012, Table 2) a potential influence cannot be ruled out. A limitation in the current study design is the correlational approach to relate behavior and resting-state dynamics. We cannot rule out that an unknown third variable may have caused the observed effects. To what extent a manipulation of the content of self-generated thoughts could affect the dynamics of functional connectivity requires further research.

Understanding the dynamics of brain (Smith et al., 2012; Hutchison et al., 2013a; Allen et al., 2014) and mental states (Smallwood, 2013) is thought to be important as it may explain the flexible way that cognition unfolds over time. We identified dynamics in network participation for brain regions that occurred at multi-second time-scales which are correlated with alterations in self-reported experience. Although traditional functional connectivity approaches have until recently often ignored fluctuations over time, our findings suggest that understanding the dynamics of cognition may depend upon methods that interrogate the complexity and flexibility of brain dynamics. Our demonstration of a relation between the dynamic variation in network participation of brain regions and psychological measures of experience, therefore, indicates that understanding the temporal brain dynamics could illuminate the processes through which different characteristics of self-generated experience arise.

\section{ACKNOWLEDGMENTS}

We like to thank Joachim Böttger and Katja Heuer for their help in visualizing connectivity graphs. 


\section{REFERENCES}

Ahn, Y. Y., Bagrow, J. P., and Lehmann, S. (2010). Link communities reveal multiscale complexity in networks. Nature 466, 761-764. doi: 10.1038/nature09182

Allen, E. A., Damaraju, E., Plis, S. M., Erhardt, E. B., Eichele, T., and Calhoun, V. D. (2014). Tracking whole-brain connectivity dynamics in the resting state. Cereb. Cortex 24, 663-676. doi: 10.1093/cercor/bhs352

Andrews-Hanna, J. R., Reidler, J. S., Sepulcre, J., Poulin, R., and Buckner, R. L. (2010). Functional-anatomic fractionation of the brain's default network. Neuron 65, 550-562. doi: 10.1016/j.neuron.2010.02.005

Avants, B. B., Tustison, N. J., Song, G., Cook, P., Klein, A., and Gee, J. C. (2011). A reproducible evaluation of ANTs similarity metric performance in brain image registration. Neuroimage 54, 2033-2044. doi: 10.1016/j.neuroimage.2010 09.025

Barron, E., Riby, L. M., Greer, J., and Smallwood, J. (2011). Absorbed in thought the effect of mind wandering on the processing of relevant and irrelevant events. Psychol. Sci. 22, 596-601. doi: 10.1177/0956797611404083

Bassett, D. S., Wymbs, N. F., Porter, M. A., Mucha, P. J., Carlson, J. M., and Grafton, S. T. (2011). Dynamic reconfiguration of human brain networks during learning. Proc. Natl. Acad. Sci. U.S.A. 108, 7641-7646. doi: 10.1073/pnas.1018985108

Beckmann, C. F., DeLuca, M., Devlin, J. T., and Smith, S. M. (2005). Investigations into resting-state connectivity using independent component analysis. Philos. Trans. R. Soc. Lond. B Biol. Sci. 360, 1001-1013. doi: 10.1098/rstb.2005.1634

Behzadi, Y., Restom, K., Liau, J., and Liu, T. T. (2007). A component based noise correction method (CompCor) for BOLD and perfusion based fMRI. Neuroimage 37, 90-101. doi: 10.1016/j.neuroimage.2007.04.042

Biswal, B. B., Mennes, M., Zuo, X.-N., Gohel, S., Kelly, C., Smith, S. M., et al. (2010). Toward discovery science of human brain function. Proc. Natl. Acad. Sci. U.S.A. 107, 4734-4739. doi: 10.1073/pnas.0911855107

Böttger, J., Schurade, R., Jakobsen, E., Schaefer, A., and Margulies, D. S. (2014). Connexel Visualization: a software implementation of glyphs and edge-bundling for dense connectivity data using brainGL. Front. Neurosci. 8:15. doi: 10.3389/fnins.2014.00015

Britz, J., Van De Ville, D., and Michel, C. M. (2010). BOLD correlates of EEG topography reveal rapid resting-state network dynamics. Neuroimage 52, 1162-1170. doi: 10.1016/j.neuroimage.2010.02.052

Buckner, R. L., Sepulcre, J., Talukdar, T., Krienen, F. M., Liu, H., Hedden, T., et al. (2009). Cortical hubs revealed by intrinsic functional connectivity: mapping, assessment of stability, and relation to Alzheimer's disease. J. Neurosci. 29, 1860-1873. doi: 10.1523/JNEUROSCI.5062-08.2009

Calhoun, V. D., Adali, T., Pearlson, G. D., and Pekar, J. J. (2001). A method for making group inferences from functional MRI data using independent component analysis. Hum. Brain Mapp. 14, 140-151. doi: 10.1002/hbm.1048

Chai, X. J., Castañón, A. N., Öngür, D., and Whitfield-Gabrieli, S. (2012). Anticorrelations in resting state networks without global signal regression. Neuroimage 59, 1420-1428. doi: 10.1016/j.neuroimage.2011.08.048

Chang, C., and Glover, G. H. (2010). Time-frequency dynamics of restingstate brain connectivity measured with fMRI. Neuroimage 50, 81-98. doi 10.1016/j.neuroimage.2009.12.011

Chang, C., Metzger, C. D., Glover, G. H., Duyn, J. H., Heinze, H.-J., and Walter, M. (2013). Association between heart rate variability and fluctuations in resting-state functional connectivity. Neuroimage 68, 93-104. doi: 10.1016/j.neuroimage.2012.11.038

Christoff, K., Gordon, A. M., Smallwood, J., Smith, R., and Schooler, J. W. (2009) Experience sampling during fMRI reveals default network and executive system contributions to mind wandering. Proc. Natl. Acad. Sci. U.S.A. 106, 8719-8724. doi: 10.1073/pnas.0900234106

Cole, M. W., Reynolds, J. R., Power, J. D., Repovs, G., Anticevic, A., and Braver, T. S. (2013). Multi-task connectivity reveals flexible hubs for adaptive task control. Nat. Neurosci. 16, 1348-1355. doi: 10.1038/nn.3470

Cox, R. W. (1996). AFNI: software for analysis and visualization of functional magnetic resonance neuroimages. Comput. Biomed. Res. 29, 162-173. doi 10.1006/cbmr.1996.0014

Craddock, R. C., James, G. A., Holtzheimer, P. E., Hu, X. P., and Mayberg, H. S. (2012). A whole brain fMRI atlas generated via spatially constrained spectral clustering. Hum. Brain Mapp. 33, 1914-1928. doi: 10.1002/hbm.21333

Cribben, I., Haraldsdottir, R., Atlas, L. Y., Wager, T. D., and Lindquist, M. A. (2012). Dynamic connectivity regression: determining state-related changes in brain connectivity. Neuroimage 61, 907-920. doi: 10.1016/j.neuroimage.2012. 03.070
Dale, A. M., Fischl, B., and Sereno, M. I. (1999). Cortical surface-based analysis: I. Segmentation and surface reconstruction. Neuroimage 9, 179-194. doi: 10.1006/nimg. 1998.0395

Damoiseaux, J. S., Rombouts, S. A. R. B., Barkhof, F., Scheltens, P., Stam, C. J., Smith, S. M., et al. (2006). Consistent resting-state networks across healthy subjects. Proc. Natl. Acad. Sci. U.S.A. 103, 13848-13853. doi: $10.1073 /$ pnas.0601417103

Deco, G., Jirsa, V. K., and McIntosh, A. R. (2011). Emerging concepts for the dynamical organization of resting-state activity in the brain. Nat. Rev. Neurosci. 12, 43-56. doi: 10.1038/nrn2961

Devinsky, O., Morrell, M. J., and Vogt, B. A. (1995). Contributions of anterior cingulate cortex to behaviour. Brain 118, 279-306. doi: 10.1093/brain/118.1.279

Dickinson, P. S. (1995). Interactions among neural networks for behavior. Curr Opin. Neurobiol. 5, 792-798. doi: 10.1016/0959-4388(95)80108-1

Garrett, D. D., Kovacevic, N., McIntosh, A. R., and Grady, C. L. (2011). The importance of being variable. J. Neurosci. 31, 4496-4503. doi: 10.1523/JNEUROSCI.5641-10.2011

Garrett, D. D., Kovacevic, N., McIntosh, A. R., and Grady, C. L. (2013). The modulation of BOLD variability between cognitive states varies by age and processing speed. Cereb. Cortex 23, 684-693. doi: 10.1093/cercor/bhs055

Gong, G., He, Y., Concha, L., Lebel, C., Gross, D. W., Evans, A. C., et al. (2009). Mapping anatomical connectivity patterns of human cerebral cortex using in vivo diffusion tensor imaging tractography. Cereb. Cortex 19, 524-536. doi: 10.1093/cercor/bhn102

Gorgolewski, K., Burns, C. D., Madison, C., Clark, D., Halchenko, Y. O., Waskom, M. L., et al. (2011). Nipype: a flexible, lightweight and extensible neuroimaging data processing framework in python. Front. Neuroinform. 5:13. doi: 10.3389/fninf.2011.00013

Gorgolewski, K. J., Lurie, D. J., Urchs S., Kipping, J. A., Craddock, R. C., Milham, M. P., et al. (in press). A correspondence between individual differences in the brain's intrinsic functional architecture and the content and form of selfgenerated thoughts. PLoS ONE.

Greve, D. N., and Fischl, B. (2009). Accurate and robust brain image alignment using boundary-based registration. Neuroimage 48, 63-72. doi: 10.1016/j.neuroimage.2009.06.060

Hagmann, P., Cammoun, L., Gigandet, X., Meuli, R., Honey, C. J., Wedeen, V. J., et al. (2008). Mapping the structural core of human cerebral cortex. PLoS Biol. 6:e159. doi: 10.1371/journal.pbio.0060159

Handwerker, D. A., Roopchansingh, V., Gonzalez-Castillo, J., and Bandettini, P. A. (2012). Periodic changes in fMRI connectivity. Neuroimage 63, 1712-1719. doi: 10.1016/j.neuroimage.2012.06.078

Hong, S. L., and Rebec, G., V (2012). Biological sources of inflexibility in brain and behavior with aging and neurodegenerative diseases. Front. Syst. Neurosci. 6:77. doi: 10.3389/fnsys.2012.00077

Hooper, S. L., and Moulins, M. (1989). Switching of a neuron from one network to another by sensory-induced changes in membrane properties. Science 244, 1587-1589. doi: 10.1126/science. 2740903

Horn, J. L. (1965). A rationale and test for the number of factors in factor analysis. Psychometrika 30, 179-185. doi: 10.1007/BF02289447

Hutchison, R. M., Womelsdorf, T., Allen, E. A., Bandettini, P. A., Calhoun, V. D., Corbetta, M., et al. (2013a). Dynamic functional connectivity: promise, issues, and interpretations. Neuroimage 80, 360-378. doi: 10.1016/j.neuroimage.2013.05.079

Hutchison, R. M., Womelsdorf, T., Gati, J. S., Everling, S., and Menon, R. S. (2013b). Resting-state networks show dynamic functional connectivity in awake humans and anesthetized macaques. Hum. Brain Mapp. 34, 2154-2177. doi: 10.1002/hbm.22058

Jenkinson, M., Beckmann, C. F., Behrens, T. E. J., Woolrich, M. W., and Smith, S M. (2012). Fsl. Neuroimage 62, 782-790. doi: 10.1016/j.neuroimage.2011.09.015

Kalinka, A. T., and Tomancak, P. (2011). linkcomm: an R package for the generation, visualization, and analysis of link communities in networks of arbitrary size and type. Bioinformatics 27, 2011-2012. doi: 10.1093/bioinformatics/btr311

Kelly, A. M. C., Uddin, L. Q., Biswal, B. B., Castellanos, F. X., and Milham, M. P. (2008). Competition between functional brain networks mediates behavioral variability. Neuroimage 39, 527-537. doi: 10.1016/j.neuroimage.2007.08.008

Lee, J.-H., Hashimoto, R., Wible, C. G., and Yoo, S.-S. (2011). Investigation of spectrally coherent resting-state networks using non-negative matrix factorization for functional MRI data. Int. J. Imaging Syst. Technol. 21, 211-222. doi: 10.1002/ima.20276 
Lohmann, G., Margulies, D. S., Horstmann, A., Pleger, B., Lepsien, J., Goldhahn, D., et al. (2010). Eigenvector centrality mapping for analyzing connectivity patterns in fMRI data of the human brain. PLoS ONE 5:e10232. doi: 10.1371/journal.pone.0010232

Majeed, W., Magnuson, M., and Keilholz, S. D. (2009). Spatiotemporal dynamics of low frequency fluctuations in BOLD fMRI of the rat. J. Magn. Reson. Imaging 30, 384-393. doi: 10.1002/jmri.21848

Mantel, N. (1967). The detection of disease clustering and a generalized regression approach. Cancer Res. 27, 209-220.

Marder, E., and Bucher, D. (2001). Central pattern generators and the control of rhythmic movements. Curr. Biol. 11, 986-996. doi: 10.1016/S09609822(01)00581-4

Marder, E., and Bucher, D. (2007). Understanding circuit dynamics using the stomatogastric nervous system of lobsters and crabs. Annu. Rev. Physiol. 69, 291-316. doi: 10.1146/annurev.physiol.69.031905.161516

McIntosh, A. R., Kovacevic, N., Lippe, S., Garrett, D., Grady, C., and Jirsa, V. (2010). The development of a noisy brain. Arch. Ital. Biol. 148, 323-337. doi: 10.1016/j.tics.2010.04.004

McQuitty, L. L. (1966). Similarity analysis by reciprocal pairs for discrete and continuous data. Educ. Psychol. Meas. 26, 825-831. doi: 10.1177/001316446602 600402

Musso, F., Brinkmeyer, J., Mobascher, A., Warbrick, T., and Winterer, G. (2010). Spontaneous brain activity and EEG microstates. A novel EEG/fMRI analysis approach to explore resting-state networks. Neuroimage 52, 1149-1161. doi: 10.1016/j.neuroimage.2010.01.093

Nooner, K. B., Colcombe, S. J., Tobe, R. H., Mennes, M., Benedict, M. M., Moreno, A. L., et al. (2012). The NKI-rockland sample: a model for accelerating the pace of discovery science in psychiatry. Front. Neurosci. 6:152. doi: 10.3389/fnins.2012.00152

Patriat, R., Molloy, E. K., Meier, T. B., Kirk, G. R., Nair, V. A., Meyerand, M. E., et al. (2013). The effect of resting condition on resting-state fMRI reliability and consistency: a comparison between resting with eyes open, closed, and fixated. Neuroimage 78, 463-473. doi: 10.1016/j.neuroimage.2013.04.013

Poldrack, R. A., and Packard, M. G. (2003). Competition among multiple memory systems: converging evidence from animal and human brain studies. Neuropsychologia 41, 245-251. doi: 10.1016/S0028-3932(02)00157-4

Power, J. D., Cohen, A. L., Nelson, S. M., Wig, G. S., Barnes, K. A., Church, J. A., et al. (2011). Functional network organization of the human brain. Neuron 72, 665-678. doi: 10.1016/j.neuron.2011.09.006

Revelle, W. (2011). Psych: Procedures for Psychological, Psychometric, and Personality Research. Evanston, IL: R Packag. Version 1019.

Ruby, F. J. M., Smallwood, J., Engen, H., and Singer, T. (2013). How selfgenerated thought shapes mood-the relation between mind-wandering and mood depends on the socio-temporal content of thoughts. PLoS ONE 8:e77554. doi: 10.1371/journal.pone.0077554

Schölvinck, M. L., Maier, A., Ye, F. Q., Duyn, J. H., and Leopold, D. A. (2010). Neural basis of global resting-state fMRI activity. Proc. Natl. Acad. Sci. U.S.A. 107, 10238-10243. doi: 10.1073/pnas.0913110107

Schwarz, A. J., and McGonigle, J. (2011). Negative edges and soft thresholding in complex network analysis of resting state functional connectivity data. Neuroimage 55, 1132-1146. doi: 10.1016/j.neuroimage.2010.12.047

Smallwood, J. (2013). Distinguishing how from why the mind wanders: a processoccurrence framework for self-generated mental activity. Psychol. Bull. 139, 519-535. doi: 10.1037/a0030010

Smallwood, J., Brown, K. S., Baird, B., Mrazek, M. D., Franklin, M. S., and Schooler, J. W. (2012). Insulation for daydreams: a role for tonic norepinephrine in the facilitation of internally guided thought. PLoS ONE 7:e33706. doi: 10.1371/journal.pone.0033706

Smallwood, J., Fitzgerald, A., Miles, L. K., and Phillips, L. H. (2009). Shifting moods, wandering minds: negative moods lead the mind to wander. Emotion 9, 271-276. doi: 10.1037/a0014855

Smallwood, J., and O'Connor, R. C. (2011). Imprisoned by the past: unhappy moods lead to a retrospective bias to mind wandering. Cogn. Emot. 25, 1481-1490. doi: 10.1080/02699931.2010.545263
Smallwood, J., O'Connor, R. C., Sudbery, M. V., and Obonsawin, M. (2007). Mind-wandering and dysphoria. Cogn. Emot. 21, 816-842. doi: 10.1080/02699930600911531

Smallwood, J., Tipper, C., Brown, K., Baird, B., Engen, H., Michaels, J. R., et al. (2013). Escaping the here and now: evidence for a role of the default mode network in perceptually decoupled thought. Neuroimage 69, 120-125. doi: 10.1016/j.neuroimage.2012.12.012

Smith, S. M., Miller, K. L., Moeller, S., Xu, J., Auerbach, E. J., Woolrich, M. W., et al. (2012). Temporally-independent functional modes of spontaneous brain activity. Proc. Natl. Acad. Sci. U.S.A. 109, 3131-3136. doi: 10.1073/pnas.1121329109

Sokal, R. R., and Rohlf, F. J. (1962). The comparison of dendrograms by objective methods. Taxon 11, 33-40. doi: 10.2307/1217208

Sporns, O., Honey, C. J., and Kötter, R. (2007). Identification and classification of hubs in brain networks. PLoS ONE 2:e1049. doi: 10.1371/journal.pone.0001049

Tagliazucchi, E., Von Wegner, F., Morzelewski, A., Brodbeck, V., and Laufs, H. (2012). Dynamic BOLD functional connectivity in humans and its electrophysiological correlates. Front. Hum. Neurosci. 6:339. doi: 10.3389/fnhum.2012. 00339

Ten Berge, J. M. F., Krijnen, W. P., Wansbeek, T., and Shapiro, A. (1999). Some new results on correlation-preserving factor scores prediction methods. Linear Algebra Appl. 289, 311-318. doi: 10.1016/S0024-3795(97)10007-6

Thompson, G. J., Magnuson, M. E., Merritt, M. D., Schwarb, H., Pan, W.J., McKinley, A., et al. (2013). Short-time windows of correlation between large-scale functional brain networks predict vigilance intraindividually and interindividually. Hum. Brain Mapp. 34, 3280-3298. doi: 10.1002/hbm. 22140

Van den Heuvel, M. P., and Sporns, O. (2013). An anatomical substrate for integration among functional networks in human cortex. J. Neurosci. 33, 14489-14500. doi: 10.1523/JNEUROSCI.2128-13.2013

Van Dijk, K. R. A., Sabuncu, M. R., and Buckner, R. L. (2012). The influence of head motion on intrinsic functional connectivity MRI. Neuroimage 59, 431-438. doi: 10.1016/j.neuroimage.2011.07.044

Weimann, J. M., and Marder, E. (1994). Switching neurons are integral members of multiple oscillatory networks. Curr. Biol. 4, 896-902. doi: 10.1016/S09609822(00)00199-8

Xu, J., Moeller, S., Strupp, J., Auerbach, E. J., Chen, L., Feinberg, D. A., et al. (2012). "Highly accelerated whole brain imaging using aligned-blipped-controlledaliasing multiband EPI," in Proceedings of the 20th Annual Meeting of ISMRM (Melbourne), 2306.

Yeo, B. T. T., Krienen, F. M., Chee, M. W. L., and Buckner, R. L. (2014). Estimates of segregation and overlap of functional connectivity networks in the human cerebral cortex. Neuroimage 88, 212-227. doi: 10.1016/j.neuroimage.2013. 10.046

Zuo, X. N., Ehmke, R., Mennes, M., Imperati, D., Castellanos, F. X., Sporns, O., et al. (2012). Network centrality in the human functional connectome. Cereb. Cortex 22, 1862-1875. doi: 10.1093/cercor/bhr269

Conflict of Interest Statement: The authors declare that the research was conducted in the absence of any commercial or financial relationships that could be construed as a potential conflict of interest.

Received: 20 December 2013; paper pending published: 09 February 2014; accepted: 18 March 2014; published online: 06 May 2014.

Citation: Schaefer A, Margulies DS, Lohmann G, Gorgolewski KJ, Smallwood J, Kiebel SJ and Villringer A (2014) Dynamic network participation of functional connectivity hubs assessed by resting-state fMRI. Front. Hum. Neurosci. 8:195. doi: 10.3389/fnhum. 2014.00195

This article was submitted to the journal Frontiers in Human Neuroscience. Copyright (C) 2014 Schaefer, Margulies, Lohmann, Gorgolewski, Smallwood, Kiebel and Villringer. This is an open-access article distributed under the terms of the Creative Commons Attribution License (CC BY). The use, distribution or reproduction in other forums is permitted, provided the original author(s) or licensor are credited and that the original publication in this journal is cited, in accordance with accepted academic practice. No use, distribution or reproduction is permitted which does not comply with these terms. 